Research Article

\title{
Multiattribute Grey Target Decision Method Based on Soft Set Theory
}

\author{
Xia Wang, Yaoguo Dang, and Diqing Hou \\ College of Economics and Management, Nanjing University of Aeronautics and Astronautics, Nanjing 211106, China \\ Correspondence should be addressed to Xia Wang; wangxia0509@163.com
}

Received 19 June 2014; Accepted 25 September 2014; Published 14 October 2014

Academic Editor: Yuji Liu

Copyright ( 2014 Xia Wang et al. This is an open access article distributed under the Creative Commons Attribution License, which permits unrestricted use, distribution, and reproduction in any medium, provided the original work is properly cited.

\begin{abstract}
With respect to the Multiattribute decision-making problems in which the evaluation attribute sets are different and the evaluating values of alternatives are interval grey numbers, a multiattribute grey target decision-making method in which the attribute sets are different was proposed. The concept of grey soft set was defined, and its "AND" operation was assigned by combining the intersection operation of grey number. The expression approach of new grey soft set of attribute sets considering by all decision makers were gained by applying the "AND" operation of grey soft set, and the weights of synthesis attribute were proved. The alternatives were ranked according to the size of distance of bull's eyes of each alternative under synthetic attribute sets. The green supplier selection was illustrated to demonstrate the effectiveness of proposed model.
\end{abstract}

\section{Introduction}

As decision-making problems in social and economic life become more and more complicated, it is hard for individual decision makers of different knowledge structure and practical experience to consider all the aspects of decision problems. Most important decisions were made with participation of several specialists in order to ensure the scientificity and rationality of decisions, which is called group decision-making [1]. In recent years great attention has been paid to studies on multiattribute decision-making problems under uncertain environment, and plenty achievements were gained $[2,3]$. In the existing decision making methods, most decision systems give the same attribute sets; that is, all the decision makers give values of evaluation schemes depending on the same attribute sets. However, decision makers in practical decision-making problems usually have different backgrounds. Because they have different knowledge structures, practical experiences and preferences, the attribute sets they considered will not totally be the same. Thus decisionmaking problems in which each decision maker considers different attribute sets have caused concerns of researchers. Literature [4] proposes a rule to combine different evaluation indexes and a method to combine weight vectors. Literature
[5] establishes a comparison method based on fuzzy soft set "AND" operation for multi-index schemes, considering the problems of different parameter sets by different decision makers. Literature [6] defines an "and/or" decision function based on soft set, and establishes an "and/or" group decision making method which doesn't use rough set and fuzzy set. As a mathematical tool to solve problems of uncertainty, soft set [7] overcomes the shortcomings of classical mathematical theory in dealing with problems of uncertainty; thus it has been widely used in practical decision problems [8]. Because of the complexity and uncertainty of practical problems, Professor Deng Julong proposes to use gray number [9] to solve problems in which object is clear extension and unclear intension. However, when the evaluation attribute value is interval grey number, literatures using soft set theory to solve these kinds of problems are not common currently.

As a new approach to solve multiattribute decisionmaking problems, grey target decision-making [9] has got great attention since it was proposed by Professor Deng Julong. Multiattribute decision-making is usually an uncertain problem and contains multiple time points; literature [10] presents a solving model which can establish time point weights depending on grey entropy and time degree and an objective function which can aggregate distance of 
bull's eyes of each time point. Literature [11] puts forward a new integrated distance of bull's eyes by integrating the space projection distance from each situation to positive and negative target center. Literature [12] constructs an English learning results evaluation model which can collect teacher's information effectively based on grey target theory. Literature [13] uses multicriteria intelligent weighting grey target decision to select resettlement area for reservoir immigrants and analyzes the factors affecting the choice.

In this paper, soft set theory was introduced into grey system theory to solve multiattribute decision-making problems in which evaluation attribute sets are different and evaluation decision-making values are interval grey numbers. A multiattribute grey target decision-making method with different attribute sets was established by combining the features of grey target decision-making, and this method was applied in the choice of green supplier to illustrate the rationality and effectiveness of proposed method.

\section{Basic Knowledge}

Definition 1 (see [9]). Grey number which has both upper bounds $\bar{a}$ and lower bounds $\underline{a}$ is referred to as interval grey number; that is, $a(\otimes) \in[\underline{a}, \bar{a}]$, where $\underline{a} \leq \bar{a}$.

Assume that $a(\otimes) \in[\underline{a}, \bar{a}]$ and $b(\otimes) \in[\underline{b}, \bar{b}]$ are interval grey number; then

$$
\begin{gathered}
a(\otimes)+b(\otimes) \in[\underline{a}+\underline{b}, \bar{a}+\bar{b}], \\
\frac{a(\otimes)}{b(\otimes)} \in\left[\min \left\{\frac{a}{\bar{b}}, \frac{a}{\bar{b}}, \frac{\bar{a}}{\underline{b}}, \frac{\bar{a}}{\bar{b}}\right\}, \max \left\{\frac{a}{\bar{b}}, \frac{a}{\bar{b}}, \frac{\bar{a}}{\underline{b}}, \frac{\bar{a}}{\bar{b}}\right\}\right] .
\end{gathered}
$$

Definition 2 (see [9]). Assume $\otimes_{1} \in[\underline{a}, \bar{a}](\underline{a}<\bar{a}), \otimes_{2} \in$ $[\underline{b}, \bar{b}](\underline{b}>\bar{b})$; then

$$
\otimes_{1} \cap \otimes_{2}=\{\xi \mid \xi \in[\underline{a}, \bar{a}],[\underline{b}, \bar{b}]\}
$$

is defined as the intersection of interval grey numbers $\otimes_{1}$ and $\otimes_{2}$.

Definition 3. Set $a(\otimes) \in[\underline{a}, \bar{a}]$ and $b(\otimes) \in[\underline{b}, \bar{b}]$ as interval grey numbers; thus the

$$
d(A, B)=2^{-1 / 2} \sqrt{(\underline{a}-\underline{b})^{2}+(\bar{a}-\bar{b})^{2}}
$$

is distance of $a(\otimes)$ and $b(\otimes)$.

Definition 4 (see [7]). Assume that $U$ is the initial universe set, $E$ is a set of parameters, and $P(U)$ is the power set of $U$ and $A \subseteq E$. A pair $(F ; A)$ is called a soft set over $U$, where $F$ is mapping given by $F: A \rightarrow P(U)$.

In practical problems, for parameter set $E$ in Definition 3, each parameter in the $E$ can be understood as an attribute probably considered by decision maker and can also be described as a certain state of attributes. $A$ is a subset of $E$, which can be viewed as attribute set considered by a decision maker. For $\forall e \in A, F(e)$ represents a set with $e$ parameter properties, and soft set $(F ; A)$ consists of set with each parameter properties of $A$.
Example 5. There are 4 patients, $U=\left\{u_{1}, u_{2}, u_{3}, u_{4}\right\}$; parameter set is $E=\left\{e_{1}, e_{2}, e_{3}, e_{4}, e_{5}, e_{6}\right\}$, and $e_{1}=$ fever, $e_{2}=$ cough and chest tightness, $e_{3}=$ cough, $e_{4}=$ physical pain, $e_{5}=$ headache, $e_{6}=$ difficulty in breathing; we wish to determine whether it is pneumonia according to each patient's condition.

Through medical diagnosis we can get that $F\left(e_{1}\right)=\left\{u_{2}\right.$, $\left.u_{3}\right\}, F\left(e_{2}\right)=\left\{u_{1}, u_{2}\right\}, F\left(e_{3}\right)=\left\{u_{2}, u_{3}, u_{4}\right\}, F\left(e_{4}\right)=\left\{u_{2}, u_{3}\right\}$, $F\left(e_{5}\right)=\left\{u_{1}, u_{3}\right\}, F\left(e_{6}\right)=\left\{u_{1}, u_{4}\right\}$. Set $A=\left\{e_{1}, e_{2}, e_{3}, e_{5}\right\}, A \subseteq$ $E$; we can know that soft set $(F, A)=\left\{\right.$ fever $=\left\{u_{2}, u_{3}\right\}$; cough and chest tightness $=\left\{u_{1}, u_{2}\right\}$; cough $=\left\{u_{2}, u_{3}, u_{4}\right\}$; headache $\left.=\left\{u_{1}, u_{3}\right\}\right\}$ according to the definition of soft set.

In actual decision-making problems, object which is clear extension and unclear intension often appears. Such object has some gray nature, so using gray numbers to describe this kind of problem is of certain practical significance. As a mathematical tool to handle uncertain problems, soft sets overcome the shortcomings of classical mathematical theory, which lacks parameterization tool. Considering this, this paper proposes the definition of grey soft set.

Definition 6. Assume that a grey number $\otimes$ is introduced on the background or universe $U, E$ is a set of parameters, and $P(U)$ is the power set of set $U$ and $A \subseteq E$. A pair $(F ; A)$ is called a grey soft set over $U$, where $F$ is mapping given by $F: A \rightarrow P(U)$

Definition 7. Assume that $(F ; A)$ and $(G ; B)$ are two grey soft sets over common universe $U$, for $\forall(a, b) \in A \times B, H(a, b)=$ $F(a) \cap G(b)$; then $(H, A \times B)$ is defined as "AND" operation of soft grey sets $(F ; A)$ and $(G ; B)$.

By Definition 7 we can see that a new grey soft set $(H, A \times$ $B)$ can be obtained by "AND" operation of soft grey sets $(F ; A)$ and $(G ; B)$ and each parameter in $(H, A \times B)$ is synthesized with parameter in $A$ and $B$, where $F(a) \cap G(b)$ represent the intersection of two grey numbers.

Example 8. A company plans to purchase product parts; it plans to select the best green supplier from the four candidates $U=\left\{u_{1}, u_{2}, u_{3}, u_{4}\right\}$; attribute set is $E=\left\{e_{1}, e_{2}\right.$, $\left.e_{3}, e_{4}, e_{5}, e_{6}\right\}$, and $e_{1}=$ Customer satisfaction, $e_{2}=$ business reputation, $e_{3}=$ environmental impact degree, $e_{4}=$ energy consumption degree, $e_{5}=$ technical level, $e_{6}=$ product performance.

Suppose two decision makers consider different attribute sets $A=\left\{e_{1}, e_{2}\right\}$ and $B=\left\{e_{5}\right\}$, and

$$
\begin{array}{r}
F\left(e_{1}\right)=\left\{u_{1}=[0.85,0.94], u_{2}=[0.81,0.89],\right. \\
\left.u_{3}=[0.83,0.88], u_{4}=[0.82,0.91]\right\}, \\
F\left(e_{2}\right)=\left\{u_{1}=[0.85,0.92], u_{2}=[0.89,0.93],\right. \\
\left.u_{3}=[0.84,0.90], u_{4}=[0.85,0.91]\right\},
\end{array}
$$




$$
\begin{aligned}
G\left(e_{5}\right)=\left\{u_{1}\right. & =[0.79,0.87], u_{2}=[0.82,0.91], \\
u_{3} & \left.=[0.81,0.88], u_{4}=[0.78,0.86]\right\},
\end{aligned}
$$

so grey soft set generated from "AND" operation between grey soft set $(F ; A)$ and $(G ; B)$ is

$$
\begin{gathered}
H\left(e_{1}^{*}=\{\text { Customer satisfaction, technical level }\}\right) \\
=\left\{u_{1}=[0.85,0.87], u_{2}=[0.82,0.89]\right. \\
\left.u_{3}=[0.83,0.88], u_{4}=[0.82,0.85]\right\} \\
H\left(e_{2}^{*}=\{\text { business reputation, technical level }\}\right) \\
=\left\{u_{1}=[0.85,0.87], u_{2}=[0.89,0.91]\right. \\
\left.u_{3}=[0.84,0.88], u_{4}=[0.85,0.86]\right\}
\end{gathered}
$$

In the actual decision-making problems, in order to integrate evaluation information of each decision maker, "AND" operation of soft grey sets is expanded according to Definition 6 . Assume that $\left(F_{1} ; E_{1}\right),\left(F_{2} ; E_{2}\right), \ldots,\left(F_{s} ; E_{s}\right)$ are grey soft set; then we get

$$
\left(H, E_{1} \times E_{2} \times \cdots E_{s}\right)=\left(F_{1} ; E_{1}\right) \cap\left(F_{2} ; E_{2}\right) \cap \cdots \cap\left(F_{s} ; E_{s}\right) ;
$$

that is, $\forall\left(e_{1}, e_{2}, \ldots, e_{s}\right) \in E_{1} \times E_{2} \times \cdots E_{s}$; then we get

$$
H\left(e_{1}, e_{2}, \ldots, e_{s}\right)=F_{1}\left(e_{1}\right) \cap F_{2}\left(e_{2}\right) \cap \cdots F_{s}\left(e_{s}\right) .
$$

\section{Multiattribute Grey Target Decision Method Based on Soft Set}

Let $U=\left\{u_{1}, u_{2}, \ldots, u_{n}\right\}$ be the scheme set, $E=\left\{e_{1}, e_{2}, \ldots, e_{m}\right\}$ is the attribute factor set, and $D=\left\{d_{1}, d_{2}, \ldots, d_{s}\right\}$ is the expert group set who participate in. $E_{p}=\left\{e_{1}^{p}, e_{2}^{p}, \ldots, e_{l_{p}}^{p}\right\}$ is an attribute set which is considered by expert $D_{p}(p=$ $1,2, \ldots, s)$, where $e_{t}^{p}$ is the $t$ th attribute which is considered by expert $D_{p}$, and $t=1,2, \ldots, l_{p}, E_{p} \subseteq E, E_{p} \subseteq E, l_{p} \leq m$. Let $\omega_{t}^{p}$ be the weight of the attribute $e_{t}^{p}$ where $\sum_{t=1}^{l_{p}} \omega_{t}^{p}=1$, $0 \leq \omega_{t}^{p} \leq 1$.

Denote $u_{i t}^{p}\left(i=1,2, \ldots, n ; t=1,2, \ldots, l_{p} ; p=1,2, \ldots, s\right)$ as the effect evaluation value of scheme $u_{i}$ under the attribute $e_{t}^{p}$ by expert $D_{p}$, and the effect evaluation value is an interval grey number; then the effect evaluation value of scheme $u_{i}$ under the attribute $e_{t}^{p}$ by expert $D_{p}$ is $u_{i t}^{p} \in\left[\underline{u}_{i t}^{p}, \bar{u}_{i t}^{p}\right](0 \leq$ $\left.\underline{u}_{i t}^{p} \leq \bar{u}_{i t}^{p}, i=1,2, \ldots, n, t=1,2, \ldots, l_{p} ; p=1,2, \ldots, s\right)$, and $U_{p}=\left(u_{i t}^{p}\right)_{n \times l_{p}}$ is the decision matrix of various alternatives by expert $D_{p}$. Considering frequently different polarity of different attributes, in order to eliminate diversity on the dimension unit and increase comparability of the effect evaluation value of each scheme under different attributes, and comparing between the attributes, decision matrix is standard. Attributes are classified as cost-type target and benefit-type target; we introduce the following grey poor transformation formula:

(a) for benefit-type target

$$
\underline{x}_{i t}^{p}=\frac{\underline{u}_{i t}^{p}-\underline{u}_{t}^{p^{\nabla}}}{\bar{u}_{t}^{p^{\Delta}}-\underline{u}_{t}^{p^{\nabla}}}, \quad \bar{x}_{i t}^{p}=\frac{\bar{u}_{i t}^{p}-\underline{u}_{t}^{p^{\nabla}}}{\bar{u}_{t}^{p^{\Delta}}-\underline{u}_{t}^{p^{\nabla}}},
$$

(b) for cost-type target

$$
\underline{x}_{i t}^{p}=\frac{\bar{u}_{t}^{p^{\Delta}}-\bar{u}_{i t}^{p}}{\bar{u}_{t}^{p^{\Delta}}-\underline{u}_{t}^{p^{\nabla}}}, \quad \bar{x}_{i t}^{p}=\frac{\bar{u}_{t}^{p^{\Delta}}-\underline{u}_{i t}^{p}}{\bar{u}_{t}^{p^{\Delta}}-\underline{u}_{t}^{p^{\nabla}}},
$$

where $\bar{u}_{t}^{p^{\Delta}}=\max _{1 \leq t \leq l_{p}}\left\{\bar{u}_{i t}^{p}\right\}, \underline{u}_{t}^{p^{\nabla}}=\min _{1 \leq i \leq n}\left\{\underline{u}_{i t}^{p}\right\}$. Set $x_{i t}^{p} \in$ $\left(\underline{x}_{i t}^{p}, \bar{x}_{i t}^{p}\right)$ as the normalized effect evaluation vector of scheme $u_{i}$ under the attribute $e_{t}^{p}$, which is interval gray number on $[0,1]$, and the normalized effect evaluation vector of scheme $u_{i}$ under the attribute sets $E_{p}$ is

$$
x_{i}^{p}=\left(\begin{array}{llll}
x_{i 1}^{p} & x_{i 2}^{p} & \cdots & x_{i l_{p}}^{p}
\end{array}\right),
$$

and then we get the normalized decision matrix of various alternatives by expert $D_{p}$

$$
X_{p}=\left[\begin{array}{cccc}
x_{11}^{p} & x_{12}^{p} & \cdots & x_{1 l_{p}}^{p} \\
x_{21}^{p} & x_{22}^{p} & \cdots & x_{2 l_{p}}^{p} \\
\cdots & \cdots & \ddots & \cdots \\
x_{n 1}^{p} & x_{n 2}^{p} & \cdots & x_{n l_{p}}^{p}
\end{array}\right] .
$$

In order to get the comprehensive effect evaluation value of each scheme, and simultaneously considering the different attribute set considered by the decision makers, according to the attribute sets $E_{p}$ considered by experts $D_{p}$ and standardization decision matrix, the evaluation information of each scheme on various attributes is expressed as in the form of grey soft sets $\left(F_{1} ; E_{1}\right),\left(F_{2} ; E_{2}\right), \ldots,\left(F_{s} ; E_{s}\right)$; according to the formula (12), we get

$$
F_{p}\left(e_{t}^{p}\right)=\left\{x_{1 t}^{p}, x_{2 t}^{p}, \ldots, x_{n t}^{p}\right\}
$$

According to Definition 6, we do "AND" operation of soft grey set $\left(F_{1} ; E_{1}\right),\left(F_{2} ; E_{2}\right), \ldots,\left(F_{s} ; E_{s}\right)$ and get new fuzzy soft sets $\left(H, E_{1} \times E_{2} \times \cdots E_{s}\right)$. Thus it can be seen that each attribute of $\left(H, E_{1} \times E_{2} \times \cdots E_{s}\right)$ was synthesized from $s$ attributes which, respectively, belong to different attribute sets $E_{1}, E_{2}, \ldots, E_{s}$ considered by different decision makers, and there are $l=$ $l_{1} \times l_{2} \times \cdots \times l_{s}$ synthetic parameters in $\left(H, E_{1} \times E_{2} \times \cdots E_{s}\right)$.

Assume $E^{*}=\left\{e_{1}^{*}, e_{2}^{*}, \ldots, e_{l}^{*}\right\}$ is synthetic attribute set, where $e_{k}^{*}$ is the $k$ st attribute in $E^{*}$, so $e_{k}^{*}$ is synthesis of attribute $e_{t_{1}}^{1}$ of $E_{1}$, attribute $e_{t_{2}}^{2}$ of $E_{2}, \ldots$, and attribute $e_{t_{s}}^{s}$ of $E_{s}$, and $\omega_{t_{1}}^{1}$ is the weight of attribute $e_{t_{1}}^{1}, \omega_{t_{2}}^{2}$ is the weight of attribute $e_{t_{2}}^{2}, \ldots$, and $\omega_{t_{s}}^{s}$ is the weight of attribute $e_{t_{s}}^{s}$; then we get

$$
\omega_{k}^{*}=\omega_{t_{1}}^{1} \times \omega_{t_{2}}^{2} \times \cdots \times \omega_{t_{s}}^{s},
$$

where $1 \leq t_{1} \leq l_{1}, 1 \leq t_{2} \leq l_{2}, 1 \leq t_{s} \leq l_{s}$. 
Theorem 9. Assume $E_{p}=\left\{e_{1}^{p}, e_{2}^{p}, \ldots, e_{l_{p}}^{p}\right\}$ is the attribute set considered by experts $D_{p}(p=1,2, \ldots, s)$ and $L_{p}=\{1,2$, $\left.\ldots, l_{p}\right\}$ is the subscript sets; then one gets

$$
\sum_{k=1}^{l} e_{k}^{*}=\sum_{t_{1} \in L_{1}} \sum_{t_{2} \in L_{2}} \cdots \sum_{t_{s} \in L_{3}} \omega_{t_{1}}^{1} \omega_{t_{2}}^{2} \cdots \omega_{t_{s}}^{s}=1 .
$$

Proof. Consider

$$
\begin{aligned}
& \sum_{k=1}^{l} e_{k}^{*}=\sum_{t_{1} \in L_{1}} \sum_{t_{2} \in L_{2}} \cdots \sum_{t_{s} \in L_{3}} \omega_{t_{1}}^{1} \omega_{t_{2}}^{2} \cdots \omega_{t_{s}}^{s} \\
& =\omega_{1}^{s} \sum_{t_{1} \in L_{1}} \sum_{t_{2} \in L_{2}} \cdots \sum_{t_{s-1} \in L_{s-1}} \omega_{t_{1}}^{1} \omega_{t_{2}}^{2} \cdots \omega_{t_{s-1}}^{s-1} \\
& +\omega_{2}^{s} \sum_{t_{1} \in L_{1}} \sum_{t_{2} \in L_{2}} \cdots \sum_{t_{s-1} \in L_{s-1}} \omega_{t_{1}}^{1} \omega_{t_{2}}^{2} \cdots \omega_{t_{s-1}}^{s-1} \\
& +\cdots+\omega_{l_{s}}^{s} \sum_{t_{1} \in L_{1}} \sum_{t_{2} \in L_{2}} \cdots \sum_{t_{s-1} \in L_{s-1}} \omega_{t_{1}}^{1} \omega_{t_{2}}^{2} \cdots \omega_{t_{s-1}}^{s-1} \\
& =\left(\omega_{1}^{s}+\omega_{2}^{s}+\cdots \omega_{l_{s}}^{s}\right) \\
& \times \sum_{t_{1} \in L_{1}} \sum_{t_{2} \in L_{2}} \cdots \sum_{t_{s-1} \in L_{s-1}} \omega_{t_{1}}^{1} \omega_{t_{2}}^{2} \cdots \omega_{t_{s-1}}^{s-1} \\
& =\sum_{t_{1} \in L_{1}} \sum_{t_{2} \in L_{2}} \cdots \sum_{t_{s-1} \in L_{s-1}} \omega_{t_{1}}^{1} \omega_{t_{2}}^{2} \cdots \omega_{t_{s-1}}^{s-1} \\
& =\omega_{1}^{s-1} \sum_{t_{1} \in L_{1}} \sum_{t_{2} \in L_{2}} \cdots \sum_{t_{s-1} \in L_{s-2}} \omega_{t_{1}}^{1} \omega_{t_{2}}^{2} \cdots \omega_{t_{s-2}}^{s-2} \\
& +\omega_{2}^{s-1} \sum_{t_{1} \in L_{1}} \sum_{t_{2} \in L_{2}} \cdots \sum_{t_{s-1} \in L_{s-2}} \omega_{t_{1}}^{1} \omega_{t_{2}}^{2} \cdots \omega_{t_{s-2}}^{s-2} \\
& +\cdots+\omega_{l_{s-1}}^{s-1} \sum_{t_{1} \in L_{1}} \sum_{t_{2} \in L_{2}} \cdots \sum_{t_{s-1} \in L_{s-2}} \omega_{t_{1}}^{1} \omega_{t_{2}}^{2} \cdots \omega_{t_{s-2}}^{s-2} \\
& =\left(\omega_{1}^{s-1}+\omega_{2}^{s-1}+\cdots \omega_{l_{s-1}^{s-1}}\right) \\
& \times \sum_{t_{1} \in L_{1}} \sum_{t_{2} \in L_{2}} \cdots \sum_{t_{s-1} \in L_{s-2}} \omega_{t_{1}}^{1} \omega_{t_{2}}^{2} \cdots \omega_{t_{s-2}}^{s-2} \\
& =\sum_{t_{1} \in L_{1}} \sum_{t_{2} \in L_{2}} \cdots \sum_{t_{s-1} \in L_{s-2}} \omega_{t_{1}}^{1} \omega_{t_{2}}^{2} \cdots \omega_{t_{s-2}}^{s-2} \\
& =\cdots=\sum_{t_{1} \in L_{1}} \omega_{t_{1}}^{1} \\
& =\omega_{1}^{1}+\omega_{2}^{1}+\cdots \omega_{l_{1}}^{1}=1 .
\end{aligned}
$$

Definition 10. If $z_{k}^{+}=\max \left\{\left(\underline{x}_{i k}+\bar{x}_{i k}\right) / 2 \mid 1 \leq i \leq\right.$ $n\}(k=1,2, \ldots, l)$, the corresponding desired value is defined as $\left[\underline{x}_{i k}^{+}, \bar{x}_{i k}^{+}\right]$, then

$$
z^{+}=\left\{z_{1}^{+}, z_{2}^{+}, \ldots, z_{l}^{+}\right\}=\left\{\left[\underline{x}_{i 1}^{+}, \bar{x}_{i 1}^{+}\right],\left[\underline{x}_{i 2}^{+}, \bar{x}_{i 2}^{+}\right], \ldots,\left[\underline{x}_{i l}^{+}, \bar{x}_{i l}^{+}\right]\right\}
$$

is called optimal effect vector of grey-target decision-making; it is also called positive bull's-eye.
Definition 11. Define

$$
\varepsilon_{i}^{+}=2^{-1 / 2} \sum_{k=1}^{l}\left[\omega_{k}^{*}\left(\underline{x}_{i k}-\underline{x}_{i k}^{+}\right)^{2}+\omega_{k}^{*}\left(\bar{x}_{i k}-\bar{x}_{i k}^{+}\right)^{2}\right]^{1 / 2} ;
$$

then $\varepsilon_{i}^{+}$is referred to as positive bull's-eye distance of scheme $u_{i}$.

Sort the programs according to the order of $\varepsilon_{i}^{+}$, and the smaller the $\varepsilon_{i}^{+}$is, the better the program is.

\section{Example Analysis}

A company plans to purchase product parts; it plans to select the best green supplier from four candidates $U=$ $\left\{u_{1}, u_{2}, u_{3}, u_{4}\right\}$; attribute set is $E=\left\{e_{1}, e_{2}, e_{3}, e_{4}, e_{5}, e_{6}\right\}$, and $e_{1}=$ customer satisfaction, $e_{2}=$ business reputation, $e_{3}$ $=$ environmental impact degree, $e_{4}=$ energy consumption degree, $e_{5}=$ technical level, $e_{6}=$ product performance (represented by failure rate of qualified products). Both the market department and technical department will participate in the decision-making, and they consider different attribute indexes, attribute set considered by market department is $A=\left\{e_{1}, e_{2}, e_{3}\right\}$ and the weight of each attribute is $\omega_{1}^{1}=0.35$, $\omega_{2}^{1}=0.35, \omega_{3}^{1}=0.3$; attribute set considered by technical department is $B=\left\{e_{4}, e_{5}, e_{6}\right\}$ and the weight of each attribute is $\omega_{1}^{2}=0.25, \omega_{2}^{2}=0.38, \omega_{3}^{2}=0.37$. Decision matrix of each department obtained through market research is as follows, where the values of some indicators are real numbers and is considered as special interval gray numbers during the calculation process,

$$
\begin{aligned}
& U_{1}=\left|\begin{array}{lll}
{[0.82,0.89]} & {[0.81,0.89]} & {[0.21,0.28]} \\
{[0.87,0.94]} & {[0.86,0.93]} & {[0.17,0.25]} \\
{[0.79,0.87]} & {[0.84,0.90]} & {[0.22,0.30]} \\
{[0.83,0.88]} & {[0.82,0.91]} & {[0.20,0.25]}
\end{array}\right|, \\
& U_{2}=\left|\begin{array}{lll}
{[0.27,0.35]} & {[0.77,0.89]} & 8.9 \\
{[0.21,0.32]} & {[0.85,0.92]} & 5.6 \\
{[0.29,0.40]} & {[0.79,0.88]} & 7.3 \\
{[0.24,0.31]} & {[0.81,0.90]} & 9.4
\end{array}\right|
\end{aligned}
$$

First, according to formulas (8) and (9), standardized the evaluation matrix for $U_{p}=\left(u_{i t}^{p}\right)_{n \times l_{p}}$ and obtain the standardization evaluate matrix of each scheme

$$
\begin{gathered}
X_{1}=\left|\begin{array}{lll}
{[0.2000,0.6667]} & {[0.0000,0.6667]} & {[0.1538,0.6923]} \\
{[0.5333,1.0000]} & {[0.4167,1.0000]} & {[0.3846,1.0000]} \\
{[0.0000,0.5333]} & {[0.2500,0.7500]} & {[0.0000,0.6154]} \\
{[0.2667,0.6000]} & {[0.0833,0.8333]} & {[0.3846,0.7692]}
\end{array}\right|, \\
X_{2}
\end{gathered}\left|\begin{array}{lll}
{[0.2632,0.6842]} & {[0.0000,0.8000]} & {[0.1316,0.1316]} \\
{[0.4211,1.0000]} & {[0.5333,1.0000]} & {[1.0000,1.0000]} \\
{[0.0000,0.5789]} & {[0.1333,0.7333]} & {[0.5526,0.5526]} \\
{[0.4737,0.8421]} & {[0.2667,0.8667]} & {[0.0000,0.0000]}
\end{array}\right| .
$$

Represent the evaluation information as grey soft sets $\left(F_{1} ; E_{1}\right),\left(F_{2} ; E_{2}\right)$ according to formula $(12)$, then operate 
TABLE 1: Attribute composition of grey soft sets $\left(F_{1} ; E_{1}\right),\left(F_{2} ; E_{2}\right)$ after "AND” operation.

\begin{tabular}{|c|c|c|c|c|c|c|c|c|c|}
\hline Synthesized attribute & $e_{1}^{*}$ & $e_{2}^{*}$ & $e_{3}^{*}$ & $e_{4}^{*}$ & $e_{5}^{*}$ & $e_{6}^{*}$ & $e_{7}^{*}$ & $e_{8}^{*}$ & $e_{9}^{*}$ \\
\hline Original attribute & $e_{1}, e_{4}$ & $e_{1}, e_{5}$ & $e_{1}, e_{6}$ & $e_{2}, e_{4}$ & $e_{2}, e_{5}$ & $e_{2}, e_{6}$ & $e_{3}, e_{4}$ & $e_{3}, e_{5}$ & $e_{3}, e_{6}$ \\
\hline
\end{tabular}

TABLE 2: Decision matrix of grey soft sets $\left(F_{1} ; E_{1}\right),\left(F_{2} ; E_{2}\right)$ after "AND” operation.

\begin{tabular}{ccccc}
\hline & $u_{1}$ & $u_{2}$ & $u_{3}$ & $u_{4}$ \\
\hline$e_{1}^{*}$ & {$[0.2000,0.6667]$} & {$[0.4211,1.0000]$} & {$[0.0000,0.5300]$} & {$[0.2667,0.6000]$} \\
$e_{2}^{*}$ & {$[0.0000,0.6667]$} & {$[0.5333,1.0000]$} & {$[0.0000,0.5300]$} & {$[0.2667,0.6000]$} \\
$e_{3}^{*}$ & {$[0.1316,0.1316]$} & {$[0.5333,1.0000]$} & {$[0.0000,0.5300]$} & {$[0.0000,0.0000]$} \\
$e_{4}^{*}$ & {$[0.0000,0.6667]$} & {$[0.4167,1.0000]$} & {$[0.0000,0.5789]$} & {$[0.0833,0.8333]$} \\
$e_{5}^{*}$ & {$[0.0000,0.6667]$} & {$[0.4167,1.0000]$} & {$[0.1333,0.7333]$} & {$[0.0833,0.8333]$} \\
$e_{6}^{*}$ & {$[0.0000,0.1316]$} & {$[0.4167,1.0000]$} & {$[0.2500,0.5526]$} & {$[0.0000,0.0000]$} \\
$e_{7}^{*}$ & {$[0.1538,0.6842]$} & {$[0.3846,1.0000]$} & {$[0.0000,0.5789]$} & {$[0.6158]$} \\
$e_{8}^{*}$ & {$[0.0000,0.6923]$} & {$[0.3846,1.0000]$} & {$[0.2500,0.5526]$} & {$[0.2667,0.7692]$} \\
$e_{9}^{*}$ & {$[0.1316,0.1316]$} & {$[0.3846,1.0000]$} & & {$[0.0000,0.0000]$} \\
\hline
\end{tabular}

TABLE 3: Weight of each attribute after combination.

\begin{tabular}{lcccccccc}
\hline Attribute & $e_{1}^{*}$ & $e_{2}^{*}$ & $e_{3}^{*}$ & $e_{4}^{*}$ & $e_{5}^{*}$ & $e_{6}^{*}$ & $e_{7}^{*}$ & $e_{8}^{*}$ \\
\hline Weight & 0.0875 & 0.133 & 0.1295 & 0.0875 & 0.133 & 0.1295 & 0.075 & 0.114 \\
\hline
\end{tabular}

"AND" operation to $\left(F_{p} ; E_{p}\right)(p=1,2)$ according to Definition 6, and obtain the new grey soft set $\left(H, E_{1} \times\right.$ $\left.E_{2}\right)$; after combination, there will be $l=3 \times 3=9$ attributes. Suppose the attribute set after combination is $E^{*}=$ $\left\{e_{1}^{*}, e_{2}^{*}, \ldots, e_{9}^{*}\right\}$, every attribute in $E^{*}$ is obtained through combination of attributes in $E_{1}$ and $E_{2}$, and the composition of each attribute by combination is shown in Table 1 . After "AND" operation of grey soft sets $\left(F_{1} ; E_{1}\right)$ and $\left(F_{2} ; E_{2}\right)$, the obtained decision matrix is shown in Table 2.

Weight $\omega_{k}^{*}$ of each attribute can be obtained after combination according to formula (13) and is shown in Table 3. is

Positive bull's-eye distance obtained through formula (16)

$z^{+}=\{[0.4211,1.0000],[0.5333,1.0],[0.5333,1.0]$,

$[0.4167,1.0000][0.4167,1.000],[0.4167,1.000]$,

$[0.3846,1.0000],[0.3846,1.0000],[0.3846,1.0000]\} ;$

obtain bull's-eye distance of each scheme through formula (17)

$$
\begin{array}{ll}
\varepsilon_{1}^{+}=0.6948, & \varepsilon_{2}^{+}=0.5649, \\
\varepsilon_{3}^{+}=0.6861, & \varepsilon_{4}^{+}=0.6741 .
\end{array}
$$

So the schemes can be ranked as $u_{2}>u_{4}>u_{3}>u_{1}$ and decision makers can take supplier $u_{2}$ into prior consideration.

In order to further illustrate the method proposed in this paper, then we will use traditional gray target decision method to solve the above problems. First, the original data is normalized; then we can obtain the bull's-eye distance of each scheme according to formulas (16)-(17): $\varepsilon_{1}^{+}=0.9892, \varepsilon_{2}^{+}=$ $0.6843, \varepsilon_{3}^{+}=0.8953, \varepsilon_{4}^{+}=0.9466$, so the schemes are ranked as $u_{2}>u_{3}>u_{4}>u_{1}$, and $u_{2}$ is the best scheme. Comparison between the two methods shows that the order between $u_{3}$ and $u_{4}$ has changed. This is because this paper has taken into account the situation in which different decision makers consider different attribute sets, and attribute values of each schemes are not simply weighting summed, but we can fuse the attribute sets considered by different specialists firstly, and then fusion attribute values are weighting summed. The change is reasonable through analysis of the original decision matrix data, and this proves that the method proposed in this paper is reasonable and effective and provides a more rational approach to multiattribute selecting problems that involves multiparty participation and decision makers consider different attribute sets.

\section{Conclusion}

Multiple attribute grey-target decision-making approach based on grey soft set is proposed to solve multiple attribute group decision-making problems with different attribute sets. According to the feature of grey system theory and soft set, the concept of grey soft sets is defined and grey decision-making model is built based on grey soft set; a new approach is provided to solve group decision-making with differences between the attribute sets. We can see from the algorithms that the synthesized attribute in this method will sharply increase as numbers of decision makers and attributes increase, which makes the use of the method restricted and this is also the problem that the author will study. However, 
the proposed method in this paper is easy to understand, simple to calculation, operable and applicable compared with existing methods.

\section{Conflict of Interests}

The authors declare that there is no conflict of interests regarding the publication of this paper.

\section{Acknowledgments}

This work was supported by National Natural Science Foundation (71071077, 71371098); the Fundamental Research Funds for the Central Universities (NC2012001); the Major Project of Emphasis Research Base for Philosophy and Social Science in Jiangsu Province (2012JDXM005); Funding of Jiangsu Innovation Program for Graduate Education (CXZZ13_0183).

\section{References}

[1] X. Zeshui, Uncertain Multiple Attribute Decision Making Methods and Application, Tsinghua University Press, Beijing, China, 2004.

[2] Z. Ligang, C. Huayou, W. Xiao et al., "Induced continuous ordered weighted averaging operators and their applications in interval group decision making," Control and Decision, vol. 25, no. 2, pp. 179-184, 2010.

[3] Y.-J. Xu and D. Li, "Approach to reaching consensus in multiple attribute group decision making," Control and Decision, vol. 25, no. 12, pp. 1810-1814, 2010.

[4] J. Song, Y. G. Dang, and C. Y. Lin, "Application of grey group decision-making model in audition," Control and Decision, vol. 26, no. 4, pp. 507-512, 2011.

[5] Y. Wu, W. H. Qiu, and P. Zhou, "Real option model under fuzzy group decision making," Control and Decision, vol. 27, no. 12, pp. 1828-1832, 2012.

[6] S. Benshan, Y. Jimei, and W. Jingye, "Researehes on joining theory and method with appraisal index sets," Journal of Southwest Jiaotong University, vol. 26, no. 3, pp. 73-79, 1991.

[7] A. R. Roy and P. K. Maji, "A fuzzy soft set theoretic approach to decision making problems," Journal of Computational and Applied Mathematics, vol. 203, no. 2, pp. 412-418, 2007.

[8] N. Çağman and S. Enginoğlu, "Soft set theory and uni-int decision making," European Journal of Operational Research, vol. 207, no. 2, pp. 848-855, 2010.

[9] N. Cagman and S. Enginoglu, "Soft matrix theory and its decision making," Computers \& Mathematics with Applications, vol. 59, no. 10, pp. 3308-3314, 2010.

[10] D. Molodtsov, "Soft set theory-first results," Computers \& Mathematics with Applications, vol. 37, no. 4-5, pp. 19-31, 1999.

[11] P. K. Maji, R. Biswas, and A. R. Roy, "Soft set theory," Computers \& Mathematics with Applications, vol. 45, no. 4-5, pp. 555-562, 2003.

[12] P. K. Maji, A. R. Roy, and R. Biswas, "An application of soft sets in a decision making problem," Computers \& Mathematics with Applications, vol. 44, no. 8-9, pp. 1077-1083, 2002.

[13] F. S. Liu, G. Y. Dang, G. Z. Fang et al., Grey System Theory and Its Application, Science Press, Beijing, China, 2010. 


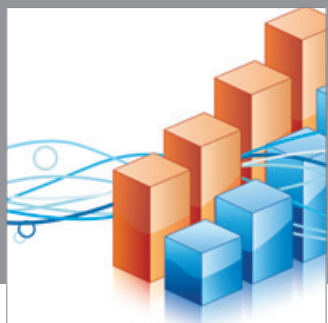

Advances in

Operations Research

mansans

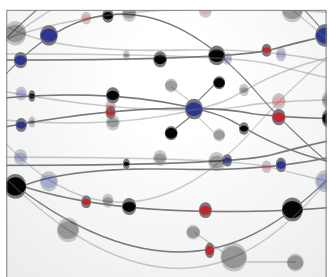

The Scientific World Journal
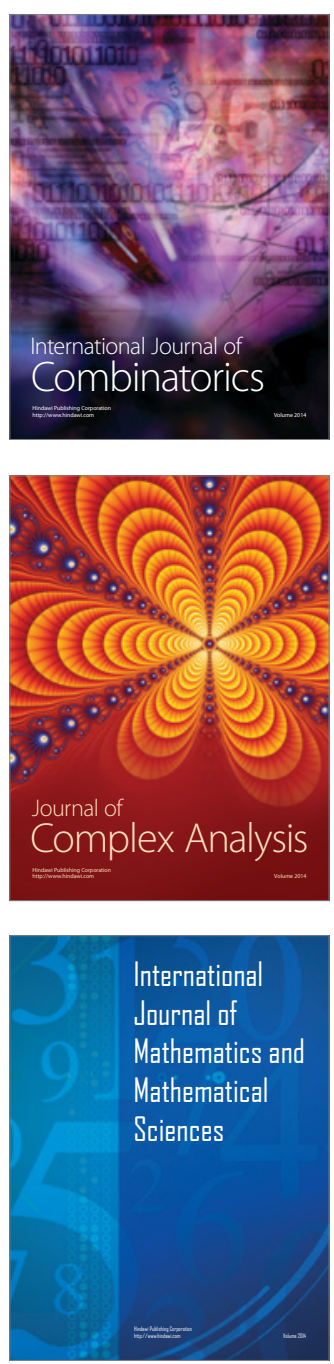
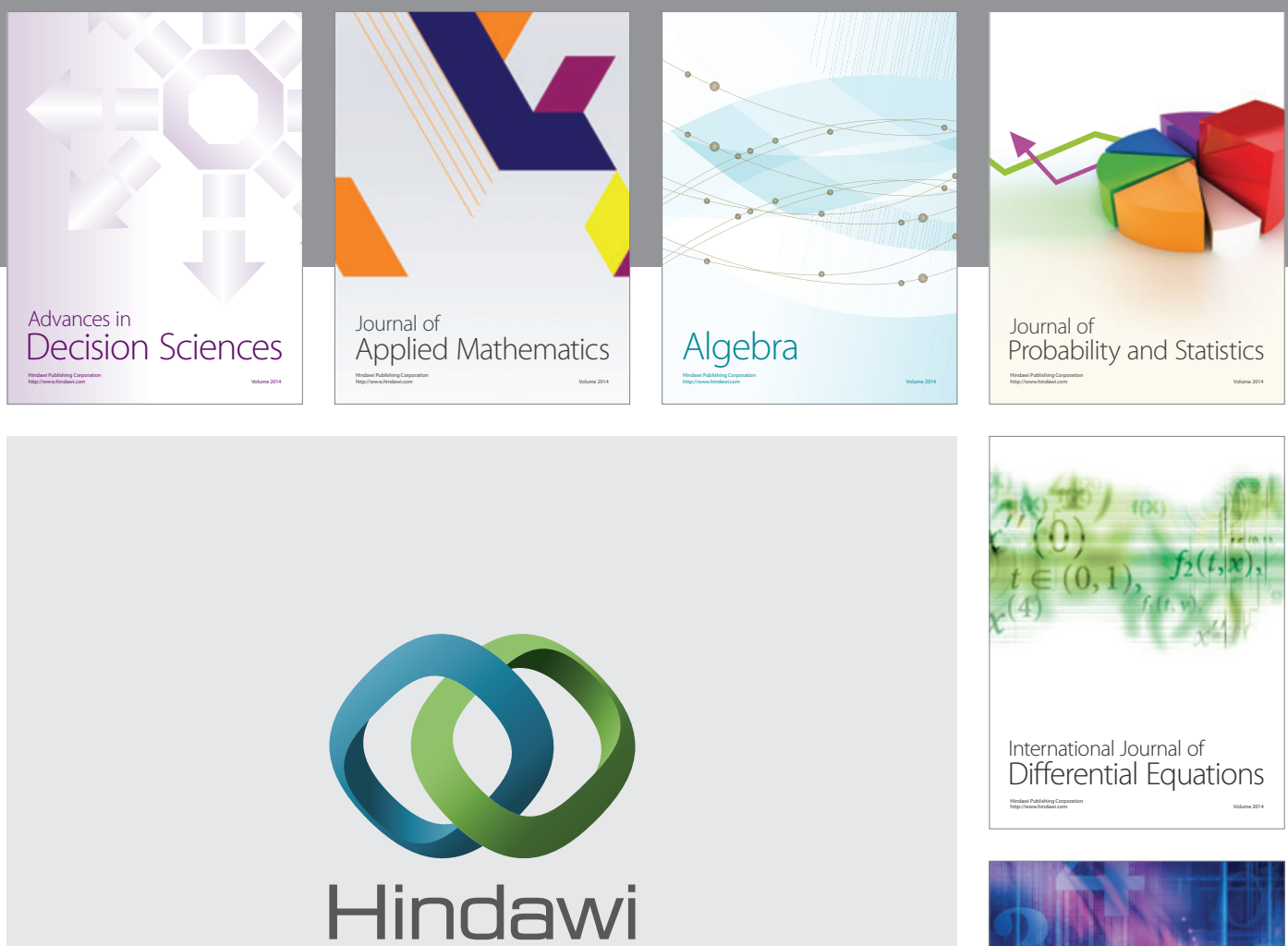

Submit your manuscripts at http://www.hindawi.com
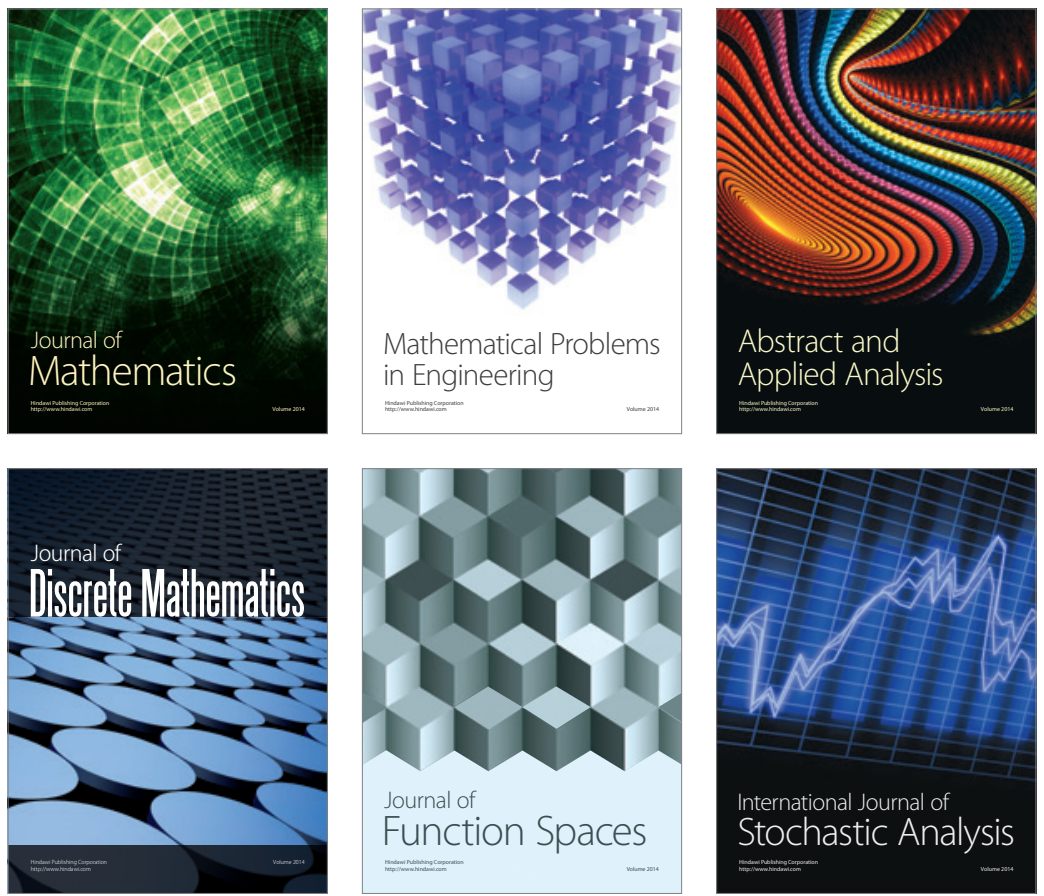

Journal of

Function Spaces

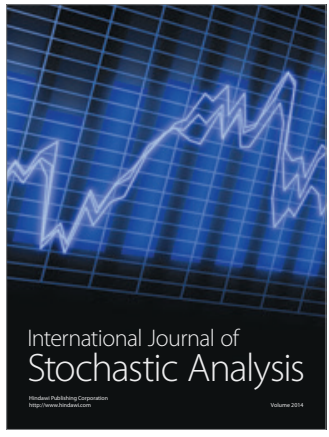

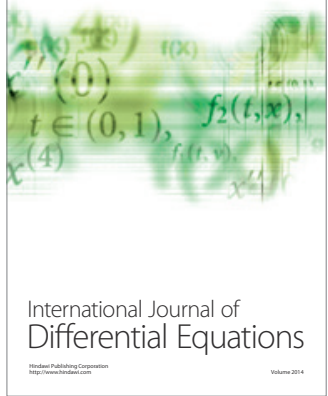
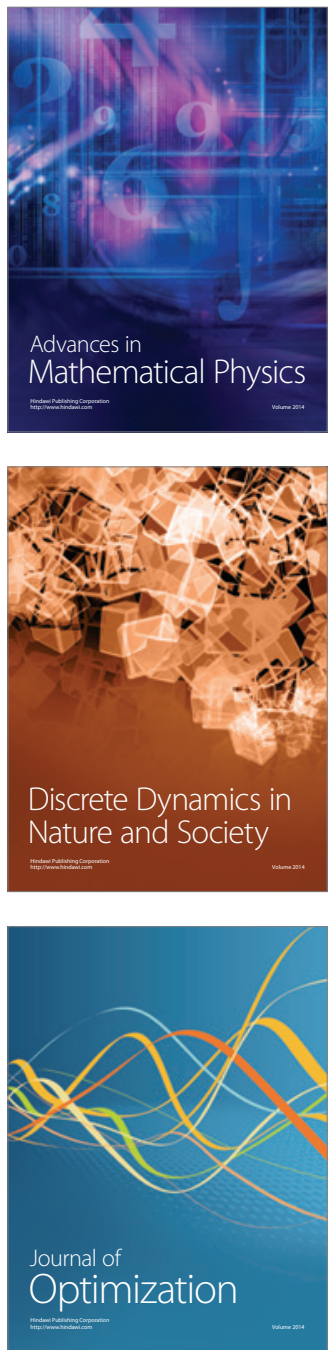